\title{
Commentary
}

\section{Systemic Viral Infections and Collateral Damage in the Liver}

\author{
David H. Adams and Stefan G. Hubscher \\ From the Liver Research Laboratories and MRC Centre for \\ Immune Regulation, Institute for Biomedical Research, Queen \\ Elizabeth Hospital, University of Birmingham, Birmingham, \\ United Kingdom
}

The liver is involved in infections by hepatotropic viruses that replicate in the liver and for which the liver is the main target. These include hepatitis $A$, hepatitis $B$, hepatitis $C$, and hepatitis $E$ viruses. In all of these infections, hepatitis and liver damage arise as a consequence of the immune response to virus within the liver. ${ }^{1}$ In addition, the liver can be affected as part of a generalized host infection with viruses that primarily target other tissues, particularly the upper respiratory tract. Examples of this phenomenon include the herpes viruses (Epstein-Barr virus, cytomegalovirus [CMV], and herpes simplex virus), parvovirus, adenovirus, ${ }^{2}$ and severe acute respiratory syndrome (SARS)-associated coronavirus. ${ }^{3}$ Liver involvement in nonhepatotropic viral infections can range from mildly deranged liver biochemistry to fulminant liver failure. In most of these infections, hepatitis is thought to be a consequence of an immune response to viral antigens with a close topographic association between the presence of viral antigens and the associated inflammatory infiltrates in the liver. Loss of immune control may be responsible for the development of hepatitis in CMV hepatitis $^{4}$ and other opportunistic viral infections such as adenovirus. ${ }^{4}$ Similar activities may also be involved in SARS-associated hepatitis, which is characterized by focal lobular lymphocytic infiltrates. ${ }^{5}$

The paper by Polakos et $\mathrm{al}^{6}$ in this issue of The American Journal of Pathology broadens the mechanism by which viruses can cause hepatitis by demonstrating that viral-specific $\mathrm{CD}^{+} \mathrm{T}$ cells, generated in response to a viral infection restricted to sites outside the liver, can trigger T-cell-mediated hepatitis in the absence of viral antigens in the liver. They describe the involvement of the liver during pulmonary infection with influenza virus and demonstrate that hepatitis can occur even in the absence of detectable virus in the liver. The authors describe the hepatitis in influenza infection as "collateral damage" and suggest that it occurs as a consequence of the recruit- ment to the liver of $\mathrm{CD}^{+}$effector $\mathrm{T}$ cells that expand systemically in response to the viral infection. These observations are of great importance for understanding the involvement of the liver in systemic infections and elucidate some of the clinical syndromes of liver inflammation that cannot be easily explained by invoking antigen-specific T-cell responses in the liver.

\section{Understanding Hepatitis in Influenza}

It is known that severe influenza infection can be associated with abnormalities in liver biochemistry that resolve after successful clearance of the virus, ${ }^{7}$ but the current study is the first to look systematically for liver involvement in volunteers infected with influenza virus. Four of 15 subjects whom Polakos et $\mathrm{al}^{6}$ infected intranasally with influenza A/Kawasaki/86 (H1N1) developed elevated serum transaminases (more than three times the upper limit in two subjects), suggesting clinically significant hepatitis. Interestingly, the rise in liver enzymes occurred after pyrexia had settled, suggesting that it was not driven by the initial viral replication and consequent activation of innate immune responses. As the authors point out, the occurrence of hepatitis in influenza is intriguing because most strains of the virus only infect the epithelial cells of the respiratory tract, and viral antigens should not therefore be present in the liver. Previous studies have shown that the liver contains a substantial population of activated $\mathrm{CD}^{+} \mathrm{T}$ cells that are specific for immunodominant epitopes in primary and secondary influenza infection and that the numbers of influenza-specific $\mathrm{CD}^{+} \mathrm{T}$ cells in the liver reflects the severity of inflammation in the lung. ${ }^{8}$ However, these studies did not make the link between the hepatic T-cell infiltrate and significant liver damage. Rather, because a high proportion of liver-infiltrating lymphocytes in influenza infection are undergoing

Accepted for publication January 11, 2006

This commentary relates to Polakos et al, Am J Pathol 2006, 11691178 , published in this issue.

Address reprint requests to David H. Adams, Liver Research Laboratories, Institute for Biomedical Research, University of Birmingham Medical School, Wolfson Dr., Birmingham, B15 2TT, UK. E-mail: d.h.adams@ bham.ac.uk. 
apoptosis, the focus was on the role of the liver in destroying antigen-specific $\mathrm{CD} 8^{+} \mathrm{T}$ cells during the resolution of the infection. ${ }^{9}$ The current paper extends these observations to demonstrate that the process of $\mathrm{CD} 8^{+}$ T-cell infiltration of the liver in influenza infection can itself lead to clinically significant hepatitis.

Due to the constraints of the clinical study, the authors were not able to correlate the development of hepatitis with the magnitude of the anti-viral $\mathrm{CD} 8^{+} \mathrm{T}$-cell response in the human volunteers. To investigate the immunopathology and kinetics of the hepatitis in more detail, they used a murine model in which both primary and secondary immune responses to influenza infection could be studied. This allowed them to show that the severity of hepatitis correlates with the magnitude of the anti-viral $\mathrm{CD}^{+} \mathrm{T}$-cell response despite the lack of detectable virus in the liver. Further experiments demonstrated that antigen-specific $\mathrm{CD}^{+}{ }^{+} \mathrm{T}$ cells were involved in the hepatitis and that neither NKT cells nor CD4 ${ }^{+} \mathrm{T}$ cells were required. Intriguingly, the hepatitis was markedly less severe in the absence of Kupffer cells, the resident macrophages of the liver. Thus, expansion of viral-specific effector $T$ cells in influenza infection can give rise to clinically significant hepatitis by a mechanism they describe as "collateral damage." The lack of antigen in the liver distinguishes this collateral damage mechanism from the previously described bystander effect, in which a mixture of antigen-specific and nonspecific effector $T$ cells mediate tissue damage at the site of infection. ${ }^{10}$

\section{Implications and Remaining Questions}

The study raises several questions. First, how and why are activated $T$ cells recruited to the liver in the absence of antigen? Previous studies have shown that activated T cells are retained in the liver in an antigen-independent manner as a consequence of interactions in the hepatic sinusoids between activated integrins on the T cell and constitutively expressed integrin ligands on sinusoidal endothelium. ${ }^{11,12}$ The trapping of activated $T$ cells in the liver is facilitated by the low flow rates and narrow caliber of the hepatic sinusoids, which promotes stochastic interactions with the rigid immunoblast as it passes through the liver. ${ }^{13}$ This mechanism would explain the unusual distribution of the lymphocytic infiltrate described by Polakos et $\mathrm{al}^{6}$, in which foci were scattered throughout the parenchyma with intervening areas of the liver parenchyma appearing to be unaffected. This would be consistent with lesions developing where activated T cells are "trapped" as a consequence of natural variations in the speed of sinusoidal flow and/or the caliber of sinusoidal vessels. This contrasts with the diffuse infiltrate of portal tracts and lobules seen when lymphocytes are responding to hepatic antigen in livers infected with hepatotropic viruses.

Second, after being trapped in the sinusoids, how do effector $\mathrm{T}$ cells mediate liver damage in the absence of their cognate antigen? One mechanism could be local ischemic necrosis precipitated by trapping of lymphocytes in the sinusoids and the consequent disturbance in blood flow. However, the nature of the lesions in the present study is against such a simple mechanism. The foci are organized into multicellular aggregates associated with hepatocyte apoptosis, suggesting that after trapping, the $T$ cells migrate across the sinusoids to interact with underlying hepatocytes. It is possible that influenza-specific T cells recognize a cross-reacting antigen on hepatocytes and trigger an "autoimmune" response. However, this seems unlikely given the fact that a similar hepatitis was seen when effector cells were expanded in response to two serologically distinct influenza viruses and to the model OVA peptide antigen SIINFEKL. Furthermore, the fact that resolution of the hepatitis paralleled resolution of the anti-viral response in the lung would argue against a classical autoimmune mechanism. It seems more likely that the recruitment of activated effector cells to the liver parenchyma results in direct activation of effector pathways by mechanisms similar to the bystander effect seen in other infections. ${ }^{10,14}$ The mechanism of hepatocyte death involves apoptosis as demonstrated by terminal deoxynucleotide transferase-mediated dUTP nick-end labeling, and activated effector $\mathrm{CD}^{+} \mathrm{T}$ cells express several receptors, including CD40 ligand and Fas-ligand, that could trigger apoptosis in hepatocytes that are particularly sensitive to Fas-mediated death. ${ }^{15,16}$ We cannot ignore the intriguing finding regarding the involvement of hepatic macrophages, Kupffer cells, in influenza hepatitis. Kupffer cells were required for the development of the lymphocytic foci but not for the recruitment of virus-specific cells to the liver. It is not clear how Kupffer cells promote hepatitis. Kupffer cells can kill hepatocytes directly via activation of Fas-dependent pathways, thereby contributing directly to local tissue damage, ${ }^{15}$ and interactions between Kupffer cells and infiltrating T cells can stimulate cytokine secretion, thereby promoting inflammation. ${ }^{17}$

Third, the study has potentially important clinical implications for liver involvement in systemic viral infections. Activation of immune responses against other extrahepatic pathogens have been shown to promote the recruitment of memory/effector cells to the liver, ${ }^{18}$ and the normal human liver contains memory $T$ cells with specificity for persistent viruses, including Epstein-Barr virus and $\mathrm{CMV},{ }^{19}$ but this does not usually lead to liver damage. By contrast in the collateral damage model proposed by Polakos et al, ${ }^{6}$ there is a pathological process leading to tissue damage. Further evidence that collateral damage in response to influenza virus may be clinically significant comes from recent reports that influenza infection can lead to exacerbation of chronic liver disease and can act as a trigger for liver allograft rejection. ${ }^{20}$ Hepatitis has been described in other respiratory infections, including respiratory syncytial virus, where its development is a poor prognostic indicator. ${ }^{21}$ Clinically significant hepatitis associated with focal lobular lymphocytic infiltrates has been reported in patients with SARS. In these cases, although SARS-associated coronavirus was detected in the liver tissues by reverse transcriptase-polymerase chain reaction, no viral particles were seen at electron microscopy. ${ }^{5}$ A recent study using a marmoset model of SARS-coronavirus infection reported a lymphocytic hep- 
atitis with histological characteristics similar to those described by Polakos et al. ${ }^{36}$ The authors were unable to demonstrate SARS viral antigen or viral RNA in hepatic tissues, and although this might have been because of technical limitations, it suggests that SARS hepatitis is also driven by collateral damage mediated by virus-specific effector cells generated in response to the pulmonary infection rather than by $T$ cells recognizing viral antigens in the liver. Alternatively, both mechanisms could operate in tandem to amplify liver damage

Finally, the collateral damage model proposed by Polakos et $\mathrm{al}^{6}$ in this issue of The American Journal of Pathology may be involved in other poorly understood forms of hepatitis. These range from the mild "nonspecific reactive hepatitis," seen in some cases of systemic febrile illness and characterized by focal parenchymal inflammatory lesions similar to those reported in the present study, to fulminant seronegative hepatitis, a rare but frequently fatal form of liver disease in which a T-cell infiltrate of the liver is associated with submassive hepatic necrosis. Searches for hepatic viruses in the latter condition have not been fruitful, and it is possible that liver damage here is also driven by collateral damage related to an extrahepatic viral infection triggering a rapid expansion of activated T cells.

\section{References}

1. Rehermann B, Nascimbeni M: Immunology of hepatitis B virus and hepatitis C virus infection. Nat Rev Immunol 2005, 5:215-229

2. Pardi DS, Romero Y, Mertz LE, Douglas DD: Hepatitis-associated aplastic anemia and acute parvovirus B19 infection: a report of two cases and a review of the literature. Am J Gastroenterol 1998, 93: $468-470$

3. Greenough TC, Carville A, Coderre J, Somasundaran M, Sullivan JL, Luzuriaga K, Mansfield K: Pneumonitis and multi-organ system disease in common marmosets (Callithrix jacchus) infected with the severe acute respiratory syndrome-associated coronavirus. Am J Pathol 2005, 167:455-463

4. Zahradnik JM, Spencer MJ, Porter DD: Adenovirus infection in the immunocompromised patient. Am J Med 1980, 68:725-732

5. Chau TN, Lee KC, Yao H, Tsang TY, Chow TC, Yeung YC, Choi KW,
Tso YK, Lau T, Lai ST, Lai CL: SARS-associated viral hepatitis caused by a novel coronavirus: report of three cases. Hepatology 2004 , 39:302-310

6. Polakos NK, Cornejo JC, Murray DA, Wright KO, Treanor JJ, Crispe IN, Topham DJ, Pierce RH: Kupffer cell-dependent hepatitis occurs during influenza infection. Am J Pathol 2006, 168:1169-1178

7. Yuen KY, Wong SS: Human infection by avian influenza A H5N1. Hong Kong Med J 2005, 11:189-199

8. Belz GT, Altman JD, Doherty PC: Characteristics of virus-specific CD8(+) T cells in the liver during the control and resolution phases of influenza pneumonia. Proc Natl Acad Sci USA 1998, 95:13812-13817

9. Crispe IN: Hepatic T cells and liver tolerance. Nat Rev Immunol 2003 3:51-62

10. Tough DF, Borrow P, Sprent J: Induction of bystander T-cell proliferation by viruses and type-i interferon in-vivo. Science 1996, 272:1947-1950

11. Mehal WZ, Juedes AE, Crispe IN: Selective retention of activated CD8 + T cells by the normal liver. J Immunol 1999, 163:3202-3210

12. Hamann A, Klugewitz K, Austrup F, Jablonski-Westrich D: Activation induces rapid and profound alterations in the trafficking of T cells. Eur J Immunol 2000, 30:3207-3218

13. Lalor PF, Shields P, Grant A, Adams DH: Recruitment of lymphocytes to the human liver. Immunol Cell Biol 2002, 80:52-64

14. Ehl S, Hombach J, Aichele P, Hengartner H, Zinkernagel RM: Bystander activation of cytotoxic T cells: studies on the mechanism and evaluation of in vivo significance in a transgenic mouse model. J Exp Med 1997, 185:1241-1251

15. Afford SC, Randhawa S, Eliopoulos AG, Hubscher SG, Young LS, Adams DH: CD40 activation induces apoptosis in cultured human hepatocytes via induction of cell surface fas ligand expression and amplifies fas-mediated hepatocyte death during allograft rejection. J Exp Med 1999, 189:441-446

16. Galle PR, Krammer PH: CD95-induced apoptosis in human liver disease. Semin Liver Dis 1998, 18:141-151

17. Knolle PA, Limmer A: Neighborhood politics: the immunoregulatory function of organ-resident liver endothelial cells. Trends Immunol $200122: 432-437$

18. Masopust D, Vezys V, Marzo AL, Lefrancois L: Preferential localization of effector memory cells in nonlymphoid tissue. Science 2001 , 291:2413-2417

19. Ward SM, Jonsson JR, Sierro S, Clouston AD, Lucas M, Vargas AL, Powell EE, Klenerman P: Virus-specific CD8+ T lymphocytes within the normal human liver. Eur J Immunol 2004, 34:1526-1531

20. Duchini A, Hendry RM, Redfield DC, Pockros PJ: Influenza infection in patients before and after liver transplantation. Liver Transpl 2000, 6:531-542

21. Eisenhut M, Thorburn K, Ahmed T: Transaminase levels in ventilated children with respiratory syncytial virus bronchiolitis. Intensive Care Med 2004, 30:931-934 